\title{
sensors
}

ISSN 1424-8220

(C) 2007 by MDPI

www.mdpi.org/sensors

Full Paper

\section{SU-8 Guiding Layer for Love Wave Devices}

\section{Paul Roach*, Shaun Atherton, Nicola Doy, Glen McHale and Michael I. Newton}

School of Science and Technology, Nottingham Trent University, Clifton Campus, Nottingham. NG11 8NS. United Kingdom

* Author to whom correspondence should be addressed; Email: Paul.Roach@ntu.ac.uk; Tel: +44(0)115-848-8062; Fax: +44(0)115-848-6616

Received: 1 October 2007 / Accepted: 31 October 2007 / Published: 1 November 2007

\begin{abstract}
SU-8 is a technologically important photoresist used extensively for the fabrication of microfluidics and MEMS, allowing high aspect ratio structures to be produced. In this work we report the use of SU-8 as a Love wave sensor guiding layer which allows the possibility of integrating a guiding layer with flow cell during fabrication. Devices were fabricated on ST-cut quartz substrates with a single-single finger design such that a surface skimming bulk wave (SSBW) at 97.4 MHz was excited. SU-8 polymer layers were successively built up by spin coating and spectra recorded at each stage; showing a frequency decrease with increasing guiding layer thickness. The insertion loss and frequency dependence as a function of guiding layer thickness was investigated over the first Love wave mode. Mass loading sensitivity of the resultant Love wave devices was investigated by deposition of multiple gold layers. Liquid sensing using these devices was also demonstrated; water-glycerol mixtures were used to demonstrate sensing of densityviscosity and the physical adsorption and removal of protein was also assessed using albumin and fibrinogen as model proteins.
\end{abstract}

Keywords: Love wave, SU-8, SU-8 guiding layer, SH-SAW

\section{Introduction}

Surface acoustic wave (SAW) devices are widely used in sensing applications with a number of variants being used in many research fields [1,2]. One of the simplest types of SAW device uses a Rayleigh wave, which propagates with an elliptical particle displacement through the substrate parallel 
to the direction of the wave. The energy of the wave is not confined to the surface, although the amplitude of the wave decays into the depth of the substrate. These types of sensors cannot be used in liquids due to the direction of mechanical displacement allowing dampening of the wave to occur. Shear horizontally polarized SAWs (SH-SAW), propagating with a particle displacement perpendicular to the wave motion, can be used in liquids but similarly are not confined to the surface of the substrate. Love waves may be propagated when an overlayer is added on the surface of a SHSAW or surface skimming bulk wave (SSBW) device. Such a wave will be supported if the guiding layer material has a shear speed less than that of the substrate [3]. An enhancement in device sensitivity can therefore be achieved, with Love waves offering one of the highest mass sensitivities. Love wave sensors have been used for a variety of applications including the detection of biological analytes [4, 5] liquid properties [6, 7] and evaporation kinetics [8].

Due to their ability to be used in an aqueous environment and their sensitivity, Love wave sensors are often used as biosensors. Different guiding layer materials have been reported such as polymethylmethacrylate (PMMA) [9, 10] positive photoresists [11] $\mathrm{SiO}_{2}$ [12] and multi-layer polymer $/ \mathrm{SiO}_{2}$ structures [13]. Although silica guiding layers display low acoustic losses in the $\mathrm{MHz}$ range their fabrication by deposition techniques is lengthy, and thick layers $(\sim 6 \mu \mathrm{m})$ are required to support a Love wave if quartz substrates are used due to the materials' similar acoustic velocities [14]. Thin polymer layers $(\sim 2 \mu \mathrm{m})$ will support a Love wave and can be spun on relatively easily. PMMA based devices exhibit lower sensitivities compared to silica, possibly due to the high internal losses occurring in the guiding layer.

One of the disadvantages of using Love wave devices is that the interdigital transducers (IDTs) are on the same side as the sensing surface. The operation of such devices in liquids can result in electrical short circuits. Harsh liquids may even damage the IDTs. For these reasons the guiding layer is often extended across the whole face of the device to avoid the use of complicated o-rings and sealed units. The use of different materials to protect the IDTs has been reported - specifically SU-8, an epoxy based negative photo resist [15]. SU-8 was used only as a protective layer to cover the IDTs whilst silicon dioxide was deposited as a guiding layer material between the IDTs. These devices were made in a multistage process, wherein silicon dioxide was grown by chemical vapour deposition, patterned between the IDTs by photolithography, with SU-8 being applied in a later process. SU-8 is spun on to a desired thickness and soft baked to remove solvent. Reactive sites within the SU-8 polymer are created during UV irradiation, which, upon heating cross-link to form a strong, hard, chemically inert surface resilient to acids, bases and solvents. [16] By using SU-8 as a guiding layer covering the IDTs any complicated and time consuming deposition of silicon dioxide wave guide layers would be reduced to a simple spin-on process. Devices would be sensitive, durable and chemically resistant.

Here we present use of SU-8 as a Love wave guiding layer material. SU-8 is assessed in terms of the thickness required to support a Love wave, with the first mode being examined. Frequency and insertion loss of the devices were monitored with increasing guiding layer thickness. The sensitivities of devices with different thickness SU-8 guiding layers were assessed by gold deposition, and liquid sensing was demonstrated using water-glycerol mixtures and protein adsorption studies. 


\section{Experimental Section}

Love wave devices were fabricated on ST-cut quartz with propagation orthogonal to the crystal direction. The IDTs consisted of sputtered gold (80 nm, Emitech) over titanium (10 nm, Emitech) as an adhesive layer, deposited using an Emitech K757X sputter coater. A single-single finger design was used. Finger widths and spacings were $12.8 \mu \mathrm{m}$, with each IDT being $4.5 \mathrm{~mm}$ in length, with an aperture of $4 \mathrm{~mm}$ and IDT centre-centre distance of $12 \mathrm{~mm}$. SU-8 is a negative photoresist requiring a multistage process to form a semi-rigid layer. Parameters were defined such that the polymer was spin coated onto a device to achieve a desired thickness, soft-baked to remove solvent before UV exposure and hard-baking to allow cross linking to take place. Once hard-baked SU-8 has the advantage over other polymers often used as a guiding layer material being mechanically reliable and almost completely chemically inert. The thickness of each layer was determined by stylus and optical profilometry, being measured at $0.56 \mu \mathrm{m}$. The SU-8 guiding layers were successively built up using SU-8-10 (Microposit) diluted in 1-methoxy-2-propanol acetate (Aldrich, 98+\%). These were spun to achieve a single layer thickness of $0.56 \mu \mathrm{m}$ (verified using a Veeco Dektak 6M stylus profiler and an optical Veeco Dektak 3 surface analysis system.) Each layer added was prebaked at $55{ }^{\circ} \mathrm{C} 10 \mathrm{~min}$, cooled to room temperature and exposed to UV using a Mega LV202E UV exposure unit, before being postbaked at $55{ }^{\circ} \mathrm{C} 10$ mins and $95{ }^{\circ} \mathrm{C} 5$ mins. Multiple $30 \mathrm{~nm}$ gold layers were used as a model for rigid mass, being sputtered on the centre of the Love wave propagation path up to $450 \mathrm{~nm}$ using an Emitech K575X fitted with a film thickness monitor. A mask was used to define a small area of $8 \mathrm{~mm}^{2}$ onto which the gold was sputtered. Device spectra were recorded using an Agilent E5061A network analyser. Glycerol/water mixtures were pumped over the sensor using a Watson Marlow peristaltic pump. Glycerol (Sigma 98\%) was diluted with distilled deionised water. Protein flow experiments were conducted with solutions being continually flown over the sensor surface at $1 \mathrm{ml} \mathrm{min}^{-1}$ by syringe pumps (Razel R100-EC). Protein solutions were prepared immediately prior to use at a concentration of $1 \mathrm{mg} \mathrm{ml}^{-1}$ in phosphate buffered saline (PBS, Sigma, tablets) using bovine serum albumin (Sigma, fraction $\mathrm{V}$, lyophilized powder) and bovine fibrinogen (Sigma, type I-S, lyophilized powder) Temperature stability of $24 \pm 0.1{ }^{\circ} \mathrm{C}$ was achieved using an Octagon 10 incubator. The Love wave device was used as the feedback element in an oscillator circuit consisting of cascaded amplifiers (Minicircuits ZFL-500LN), a $50 \mathrm{MHz}$ high pass filter and $150 \mathrm{MHz}$ low pass filter (Minicircuits BHP50 and BPL-150), a power splitter (M/A com T1000) and a frequency counter (Agilent 53132A) interfaced to a microcomputer.

\section{Results and Discussion}

Signal characteristics of devices were recorded during the build up of successive layers of SU-8 photoresist. As the guiding layer thickness increases it is expected that the acoustic wave will gradually move from propagating entirely in the quartz as a surface skimming bulk wave (SSBW) to being confined at the surface of the substrate or travelling within the guiding layer as a Love wave. As this happens the velocity of the wave will decrease and so it is expected that a decrease in resonant frequency will also occur. The additional mass loading caused by the guiding layer will also contribute to a drop in frequency. Trends in frequency and amplitude of the signal over the first mode are shown in Figure 1. The signal attenuation initially decreased with increasing guiding layer thickness, reaching 
a minimum of $-15 \mathrm{~dB}$ at $\sim 1 \mu \mathrm{m}(\mathrm{z}=0.02)$. An increase in the amplitude of the signal demonstrates that more of the acoustic energy is located near the surface of the device which is consistent with previous reports using quartz substrates [11]. Additional layers of SU-8 gave rise to a gradual decrease in insertion loss due to the dampening of the overlayer, although it is well known that higher order modes can be observed with increasing thickness $[3,11]$.

\section{Dimensionless Parameter z}

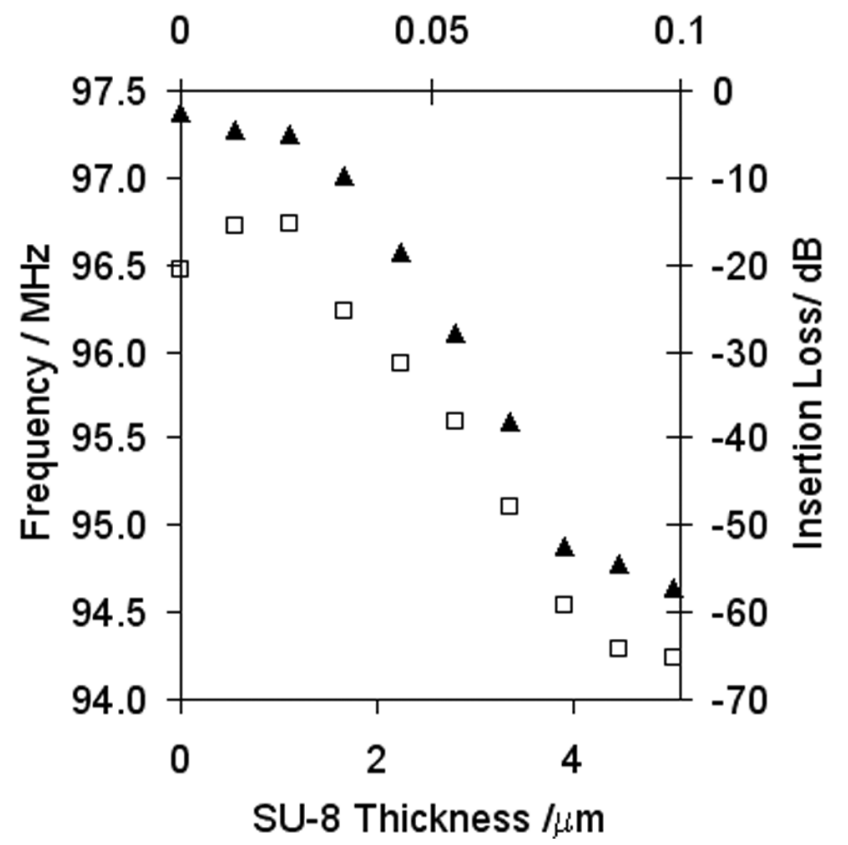

Figure 1. Signal characteristics covering the first mode of an SU-8 guiding layer

Love wave device. Frequency (closed triangles) and insertion loss (open squares) shown as a function of guiding layer thickness and dimensionless parameter $\mathrm{z}=d / \lambda_{\text {IDT }}$.

The mass sensitivity of devices with a range of guiding layer thicknesses chosen to cover the first mode has been investigated. For acoustic wave sensors without a guiding layer it usual to assume that the frequency change resulting from the addition of rigid mass is directly related to a change in phase velocity and hence a Sauerbrey type relation exists. For guided waves there may be significant dispersion and the frequency dependence of rigid mass loading onto a Love wave device may not be directly proportional [3, 17]. However if upon the addition of mass the change in guiding layer thickness is infinitesimal such that the operating point is not moved significantly down the dispersion curve, as in our experiments, then a Sauerbrey type proportionality may be observed [18]. If this is assumed then the mass loading effects of metalized layers on top of a guiding layer provide a simple route to defining the sensitivity of Love wave devices. All devices showed a linear relationship between frequency change as a function of increasing mass of gold, as shown in Figure 2. 


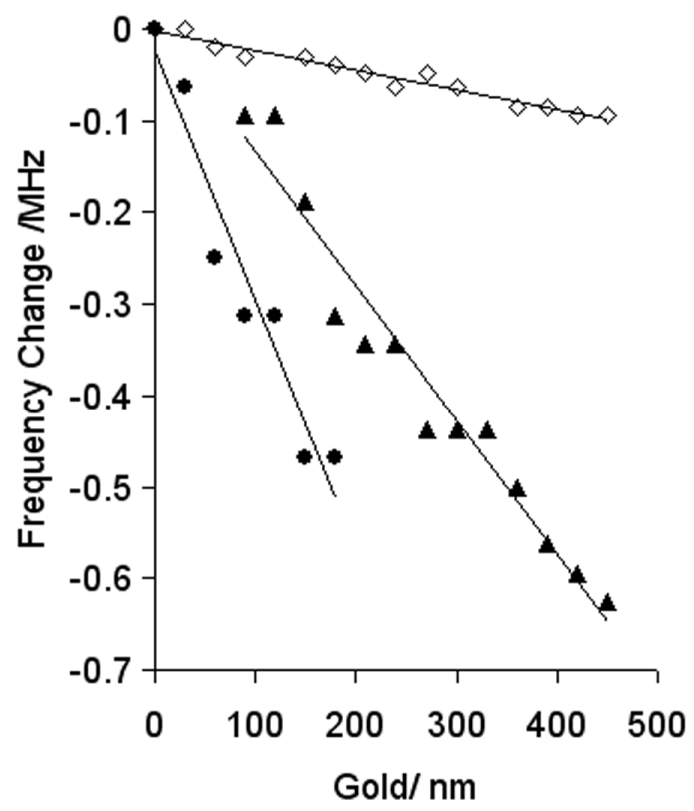

Figure 2. Relationship between mass loading and frequency of a SSBW device (open diamonds), and Love wave devices having guiding layers $1.12 \mu \mathrm{m}$ thick (closed triangles) and $2.24 \mu \mathrm{m}$ (closed circles).

A greater change in frequency was observed when depositing gold layers on all SU-8 devices compared to an uncoated device. The linear regions of the signal change was used to calculate the sensitivity of the devices in terms of a frequency change observed for mass loading of gold per unit area, Figure 3. From Figure 1 it would be expected that the most sensitive region would be where small changes in guiding layer thickness cause the largest changes in frequency, i.e. the middle of the mode. Indeed a sharp maximum in sensitivity was measured at $\sim 350 \mathrm{~Hz} / \mathrm{ng} / \mathrm{mm}^{2}$ at a guiding layer thickness of $\sim 2 \mu \mathrm{m}(\mathrm{z}=0.04)$, Figure 3 . This corresponds well with the experimental dispersion curve data (Figure 1), relating to a point where the maximum frequency changes are observed with increasing guiding layer thickness.

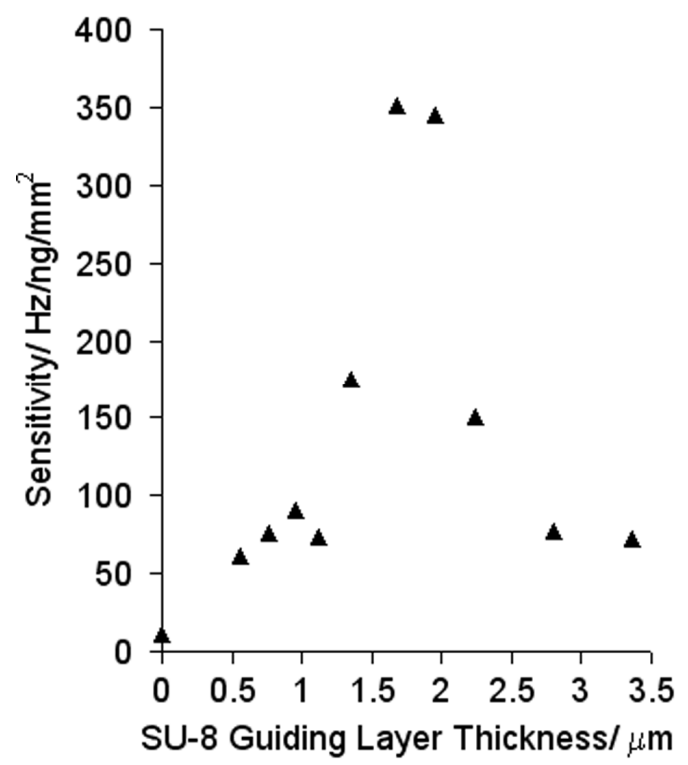

Figure 3. Frequency sensitivity of Love wave devices. 
To demonstrate the use of SU-8 Love wave devices in liquid sensing, flow experiments were conducted to compare an SSBW device to a Love wave device with a $2 \mu \mathrm{m}$ thick SU-8 guiding layer. Devices were clamped such that glycerol-water mixtures could be in contact with their sensing surface. Liquid was injected into the flow cell using a peristaltic pump with the flow direction perpendicular to the acoustic wave propagation; after the liquid was introduced the pump was halted until the next concentration was introduced. A stable signal was achieved oscillating in water, changing the liquid for $30 \%$ and $50 \%$ glycerol solutions before washing again with water, Figure 4.

The propagating acoustic waves' amplitude is attenuated by the liquid overlayer such that it decays with a penetration depth $\delta=\sqrt{\eta_{l} /\left(\pi f_{0} \rho_{l}\right)}$, where $\rho_{l}$ and $\eta_{l}$ are the liquid density and viscosity respectively. For non-guided acoustic waves the Kanazawa and Gordon relationship [19] between frequency change and square root of the density-viscosity product is assumed to hold. For Love wave devices this may not be assumed due to the considerations discussed previously for mass loading. However, again, if the operating point of the sensor is not moved significantly down the dispersion curve then proportionality between the square root of the density-viscosity product and the change in frequency may be observed.

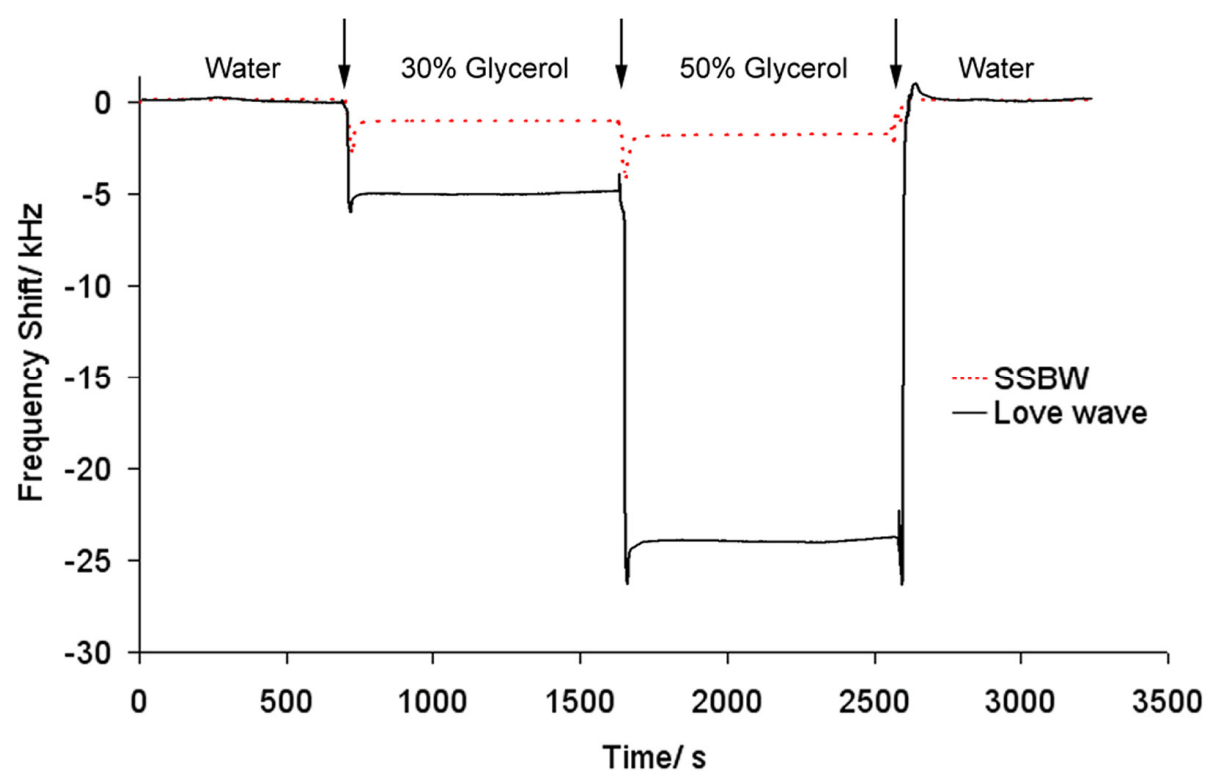

Figure 4. Liquid sensing of an SSBW device (dotted red line) and a Love wave device with an SU-8 guiding layer (solid black line), using water-glycerol mixtures.

Arrows indicate points when liquid in contact with the sensor was exchanged.

As the device is oscillated in more viscous liquids the operating frequency will decrease, shown by the stepwise drops in frequency in Figure 4 as the glycerol fraction was increased. Both the devices tested returned to their original oscillating frequency when water is replaced over the sensors, indicating that no residual glycerol remained or any changes occurred to the sensor surfaces. The Love wave device shows a much higher sensitivity compared to the SSBW device, giving frequency shift more than 4 fold and 12 fold greater than the SSBW device for 30\% and 50\% glycerol solutions respectively (relative to water). 
Initial spikes in frequency were observed due to the peristaltic pumping motion of the liquid when flushed over the crystal (causing pressure waves). Thermal drifting is often a problem during liquid sensing, wherein even small changes in temperature alter the viscosity of the media and effect the sensor itself. However, the SU-8 Love wave device showed good stability when in contact with waterglycerol mixtures, with only slight frequency drift of $\sim 11 \mathrm{~Hz} \mathrm{~min}^{-1}$. The greater stability of SU-8 compared to PMMA when in contact with water has previously been reported [20]. As SU-8 is highly chemically inert [16], it is anticipated that such devices will be stable under most chemical conditions. This may be particularly useful when measuring properties of corrosive or easily contaminated liquids such as ionic liquids.

To demonstrate the use of SU-8 Love wave devices for the detection of biomolecules, the adsorption of two serum proteins was examined. Bovine serum albumin (BSA) is a small globular protein and fibrinogen is a much larger rod-like protein, both found in high abundance in serum and are often used as model proteins in adsorption studies.[21] PBS was flown over a Love wave device having a $2 \mu \mathrm{m}$ thick SU-8 guiding layer before the inlet of protein solution. Frequency shifts observed suggest that both albumin and fibrinogen adsorb onto the SU-8 surface upon injection over the sensor (Figure 5, point a). Following adsorption any loosely adhered or unbound protein was removed during a PBS rinse (figure 5, point $b$ ) which is shown by a slight upward shift in frequency. Albumin adsorption gave rise to a much lower change in frequency $(\sim 370 \mathrm{~Hz})$ compared to fibrinogen $(\sim 1330 \mathrm{~Hz})$ which is expected due to its smaller mass and differences in surface chemistry. Fibrinogen was also found to adsorb at a faster rate. These observed trends in adsorption compare well with those previously reported for these two proteins, bearing in mind the wettability of SU-8 being neither highly hydrophilic nor hydrophobic (water contact angle $80^{\circ}$ [22]). A more detailed discussion of protein adsorption profiles and how this relates to protein-surface interactions can be found elsewhere [21].

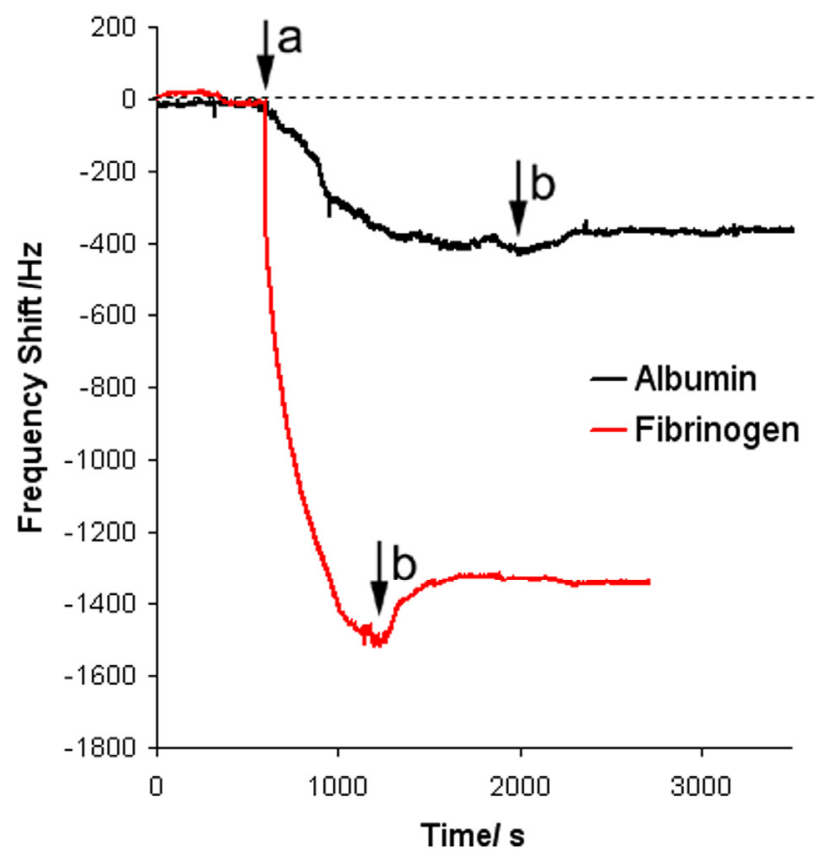

Figure 5. Protein detection using an SU-8 Love wave device. Arrows indicate points when solutions over sensors were exchanged, a) protein solution injected, prior to a) and at b) PBS solutions injected. Adsorption of albumin (black line) and fibrinogen (red line) was detected. 


\section{Conclusions}

A negative epoxy-based photoresist, SU-8, was investigated as a Love wave guiding layer material. Signal characteristics were investigated in terms of guiding layer thickness. Observed trends in frequency and insertion loss were as expected for quartz Love wave devices. Frequency decreased with increasing guiding layer thickness, whilst insertion loss reached a minimum of $\sim-15 \mathrm{~dB}$. Such low insertion losses are not common for these devices and may relate to the acoustic properties of SU-8. Sensitivity was assessed by the deposition of gold layers between the IDTs, showing a linear response in terms of frequency for all devices tested. A maximum sensitivity was achieved with a guiding layer thickness of $\sim 2 \mu \mathrm{m}(\mathrm{z}=0.04)$. Liquid sensing was also tested using water-glycerol mixtures, demonstrating the enhanced sensitivity of the Love wave device over an SSBW. Acoustic wave sensors are often used to assess viscosity-density of liquids although are often limited to those chemicals which will not damage the guiding layer The excellent stability of the devices when in contact with liquid highlights the applicability of SU-8 Love wave devices for sensing chemicals which may corrode/ dissolve/ swell or otherwise distort other polymers used as guiding layers.. The chemical stability of hard-baked SU-8 has been used by others to protect IDTs whilst another material is used as a wave guide. Protein adsorption experiments show the applicability of these sensors to detect biomolecules, showing a comparably larger frequency shift for fibrinogen adsorption than albumin and also a faster rate of adsorption for fibrinogen as expected. Here we have demonstrated the ease of fabrication and effectiveness of SU-8 based Love wave devices.

\section{Acknowledgements}

This work was funded by EPSRC grant EP/C536630/1.

\section{References and Notes}

1. Thompson, M.; Stone, D. Surface Launched Acoustic Wave Sensors: Chemical Sensing and Thin Film Applications, John Wiley and Sons: New York, 1997.

2. Lucklum, R.; Hauptmann, P. Acoustic Microsensors - The Challenge Behind Microgravimetry. Anal. Bioanal. Chem. 2006, 384(3), 667-682.

3. McHale, G.; Generalized Concept of Shear Horizontal Acoustic Plate Mode and Love Wave Sensors. Meas. Sci. Technol. 2003, 14, 1847-1853.

4. Melzak, K.A.; Ellar, D.J.; Gizeli, E. Interaction of Cytolytic Toxin CytB with a Supported Lipid Bilayer: Study Using an Acoustic Wave Device. Langmuir 2004, 20(4), 1386-1392.

5. Schlensog, M.D.; Gronewold, T.M.A.; Tewes, M.; Famulok, M.; Quandt, E. A Love-Wave Biosensor Using Nucleic Acids as Ligands. Sens. Actuators B 2004, 101(3), 308-315.

6. Melzak, K.A.; Martin, F.; Newton, M.I.; McHale, G.; Gizeli, E. Acoustic Determination of Polymer Molecular Weights and Rotation Times. J. Polymer Sci. B 2002, 40(14), 1490-1495.

7. Turton, A.; Bhattacharyya, D.; Wood, D. Liquid Density Analysis of Sucrose and Alcoholic Beverages using polyimide Guided Love-mode Acoustic Wave Sensors. Meas. Sci Technol. 2006, 17, 257-263. 
8. Razan, F.; Rebiere, D.; Dejous, C.; Monin, D.; Joanicot, M.; Conedera, V. Determination of Menthol Kinetic Constants with Love-Wave Device. Sens. Actuators B 2006, 118(1-2), 368373.

9. Gizeli, E.; Lowe, C.R.; Liley, M.; Vogel, H. Detection of Supported Lipid Layers With the Acoustic Love Waveguide Device: Application to Biosensors. Sens. Actuators B 1996, 34, 295-300.

10. Harding, L.G.; Du, J. Design and Properties of Quartz-Based Love Wave Acoustic Sensors Incorporating Silicon Dioxide and PMMA Guiding Layers. Smart Mater. Struct. 1997, 6, 716720.

11. Newton, M.I.; McHale, G.; Martin, F. Experimental Study of Love Wave Devices With Thick Guiding Layers. Sens. Actuators A 2004, 109, 180-185.

12. Jakoby, B.; Ismael, G.M.; Byfiled, M.P.; Vellekoop, M.J. A Novel Molecularly Imprinted Thin Film Applied to a Love Wave Gas Sensor. Sens. Actuators A 1999, 76, 93-97.

13. Du, J.; Harding, L.G. A Multilayer Structure for Love-Mode Acoustic. Sens. Actuators A 1998, 65, 152-159

14. Du, J.; Harding, G.L.; Ogilvy, J.A.; Dencher, P.R.; Lake, M. A Study of Love-Wave Acoustic Sensors. Sens. Actuators A 1996, 56, 211-219.

15. Arana, N.; Puente, D.; Ayerdi, I.; Castano, E.; Berganzo, J. SU8 Protective Layers in Liquid Operating SAWs. Sens. Actuators B 2006, 118, 374-379.

16. LaBianca, N.; Gelorme, J.D. High aspect ratio resist for thick film applications. Proc. SPIE, 1995, 2438, 846-852

17. McHale, G.; Newton, M.I.; Martin, F. Theoretical Mass, Liquid, and Polymer Sensitivity of Acoustic Wave Sensors With Viscoelastic Guiding Layers. J. Appl. Phys. 2003, 93(1), 675690.

18. Sauerbrey, G. The Use of Quartz Oscillators for Weighing Thin Layers and for Microweighing. Z. Phys. 1959, 155, 206-222.

19. Kanazawa, K.K.; Gordon, J.G. The Oscillation Frequency of a Quartz Resonator in Contact With a Liquid. Anal. Chim. Acta. 1985, 175, 99-105.

20. Hossenlopp, J.; Jiang, L.; Cernosek, R.; Josse, F. Characterisation of Epoxy Resin (SU-8) Film Using Thickness-Shear Mode (TSM) Resonator Under Various Conditions. J. Polymer Sci. B 2004, 42, 2373-2384.

21. Roach, P.; Farrar, D.; Perry, C.C. Interpretation of Protein Adsorption: Surface-Induced Conformational Changes. J. Am. Chem. Soc. 2005, 127, 8168-8173.

22. Shirtcliffe, N.J.; Aqil, S.; Evans, C.; McHale, G.; Newton, M.I.; Perry, C.C.; Roach, P. The use of high aspect ratio photoresist (SU-8) for super-hydrophobic pattern prototyping. $J$. Micromech. Microeng. 2004, 14, 1384-1389.

(C) 2007 by MDPI (http://www.mdpi.org). Reproduction is permitted for noncommercial purposes. 